\title{
Development of Static Var Compensator for San-yo Shinkansen
}

\author{
Keiji KAWAHARA \\ Assistant Senior Researcher, \\ Yasuji HISAMIZU \\ Assistant Senior Researcher, \\ Power Supply Systems, Power Supply Technology Development Div. \\ Yoshifumi MOCHINAGA \\ General Manager, \\ Power Supply Technology Development Div.

\begin{abstract}
This paper describes the application of SVC (Static Var Compensator) for the AC electric railway system, outlines the SVC installed at the Shin-syo sectioning post of San-yo Shinkansen and reports the results of a verification site investigation. A single-phase SVC can reduce the voltage drop in the feeding circuit and improve the power factor at substations by compensating for the reactive power. To compensate for the voltage drop, it is more effective to locate an SVC at a sectioning post than at a substation. The site investigation has revealed that an SVC can reliably suppress voltage drop.
\end{abstract}

Keywords : AC electric railway system, Static Var Compensator, San-yo Shinkansen

\section{Introduction}

In Japan, an AC electric railway system with singlephase and commercial frequency is adopted on main lines such as Shinkansen. The allowance range of feeding voltage is 22.5 to $30 \mathrm{kV}$ for Shinkansen and 16 to $22 \mathrm{kV}$ for narrow-gauge lines.

In recent years, the service load has tended to increase with an increased operation of trains, an increased speed and multiplied power. As the load capacity of a train becomes larger, the reactive power of load causes a larger voltage drop. It is assumed that the feeding voltage would drop below the lower boundary of the above-mentioned range.

The voltage drop used to be compensated for by canceling the reactance of the feeding transformer by using series capacitors or by controlling the tap-changing system of step-up transformer with thyristors, which is called the AC line voltage regulator, or ACVR for short.

With the advancement of power electronics technology, the voltage drop in feeding lines and the voltage fluctuation on the 3-phase side can now be reduced, and the power factor at substations can be improved by compensating for the reactive power by using a single-phase static var compensator, or SVC for short.

In the case of compensating for the voltage drop over the AC feeding system, SVC should be located at a sectioning post, which is at the end of the feeding circuit. The capacity of SVC can be reduced to half when it is located at a sectioning post instead of at a substation.

There are several control methods for SVC. One is continuous control using a combination of fixed capacitors with a thyristor-controlled reactor, or $\mathrm{TCR}^{1)}$ for short. Another is a stepwise control for using a thyristorswitched capacitor, TSC ${ }^{2) 344)}$ for short. Each method, which is featured by its own characteristics, is properly used while considering the actual situation of line sections.

This paper describes the principle of the voltage compensation using SVC, outlines SVC with TSC installed at the Shin-syo sectioning post of West Japan Railway Company and summarizes the results of a site investigation.

\section{Principle of voltage compensation by SVC at sectioning post}

\subsection{Principle of voltage compensation by SVC}

The voltage drop occurs at the load point due to the load current through the impedance $(Z=R+\mathrm{j} X)$ of feeding circuit as shown in Fig.1.



Fig. 1 Basic circuit of the feeding circuit using single-phase SVC at sectioning post 


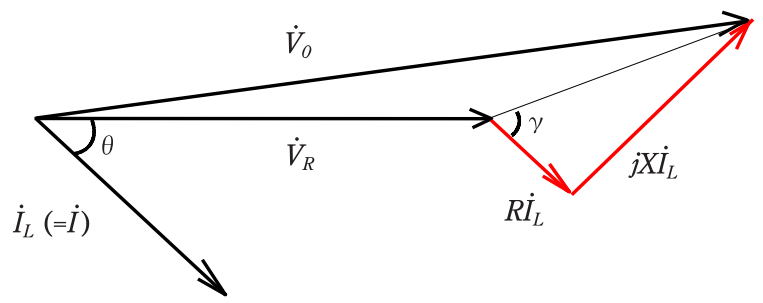

(a) Vector diagram without SVC

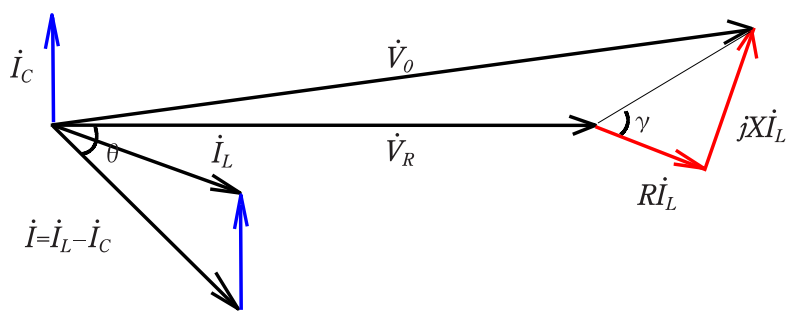

(b) Vector diagram of compensation by SVC

Fig. 2 Principle of voltage compensation using SVC

Table 1 Comparison of TSC with TCR

\begin{tabular}{|l|c|c|}
\hline & TSC & TCR \\
\hline Method of control & Stepwise & Continuous \\
\hline Withstanding voltage of thyristor & Double SVC voltage & SVC voltage \\
\hline Power losses under uncompensation & None & Large \\
\hline Generation of harmonics & None & Much \\
\hline Composition for compensation & Capacitor & Capacitor+Reactor \\
\hline
\end{tabular}

Vector diagrams of compensation by SVC are given in Fig.2. The voltage drop ratio $\varepsilon(\%)$ is expressed as follows.

$$
\varepsilon=\frac{V_{0}-V_{R}}{V_{0}} \times 100
$$

where $V_{0}$ represents the voltage at the load point without a load current and $V_{R}$ with a load current. When the phase angle between $V_{0}$ and $V_{\mathrm{R}}$ is very small, eq(1) is rewritten as follows.

$$
\begin{aligned}
\varepsilon & =\frac{I_{L} \times Z}{V_{0}} \times \cos (\theta-\gamma) \times 100 \\
& =\frac{I_{L} \times Z}{V_{0}} \times(\cos \gamma \cos \theta+\sin \gamma \sin \theta) \times 100 \\
& =\frac{I}{V_{0}^{2}} \times\left(P_{L} R+Q_{L} X\right) \times 100
\end{aligned}
$$

where

$$
\left\{\begin{array}{l}
Z=\sqrt{R^{2}+X^{2}} \\
\gamma=\tan ^{-1} \frac{X}{R} \\
P_{L}=V_{0} I_{L} \cos \theta \\
Q_{L}=V_{0} I_{L} \sin \theta
\end{array}\right.
$$

where $Z$ represents the absolute value of line impedance; $R$, resistance component; $X$, reactance component; and $P_{\mathrm{L}}$, active power.

$R$ and $X$ are determined by the feeding circuit and $P_{\mathrm{L}}$ is determined uniquely by the load. Thus we can control the reactive power $Q_{\mathrm{L}}$ to suppress the voltage drop. When SVC at the sectioning post generates reactive power $Q_{\mathrm{C}}$, $\mathrm{eq}(2)$ is rewritten as follows.

$$
\varepsilon=\frac{1}{V_{0}^{2}} \times\left\{P_{L} R+\left(Q_{\mathrm{L}}-Q_{C}\right) X\right\} \times 100
$$

When SVC generates reactive power $Q_{\mathrm{C}}$ expressed by eq(4), the voltage drop at the load point becomes zero theoretically.

$$
Q_{C}=Q_{L}+\frac{R}{X} P_{L}
$$

\subsection{Comparison between TSC and TCR}

Table 1 compares TSC with TCR. Each method with its own characteristics is adopted properly while considering the actual situation of line sections.

\section{Location and capacity of SVC}

Fig.3 illustrates the calculation results of the voltage at load point. The calculation simulates the San-yo Shinkansen. Table 2 shows parameters used in the calculation.

The SVC at the sectioning post can compensate for the voltage over the feeding circuit equally. To keep the feeding voltage over $25 \mathrm{kV}$, the rated capacity of SVC at the substation needs to be at least 27MVA. On the other hand, the capacity of the SVC at the sectioning post needs to be only 20MVA. Therefore, it is confirmed that the

Table 2 Conditions of calculation

\begin{tabular}{|l|c|}
\hline Impedance of power source & $\mathrm{j} 0.36 \Omega$ \\
\hline Impedance of feeding transform & $0.09+\mathrm{j} 1.55 \Omega$ \\
\hline Impedance of feeding circuit & $0.19 \Omega / \mathrm{km} \angle 76^{\circ}$ \\
\hline Feeding distance & $22 \mathrm{~km}$ \\
\hline
\end{tabular}



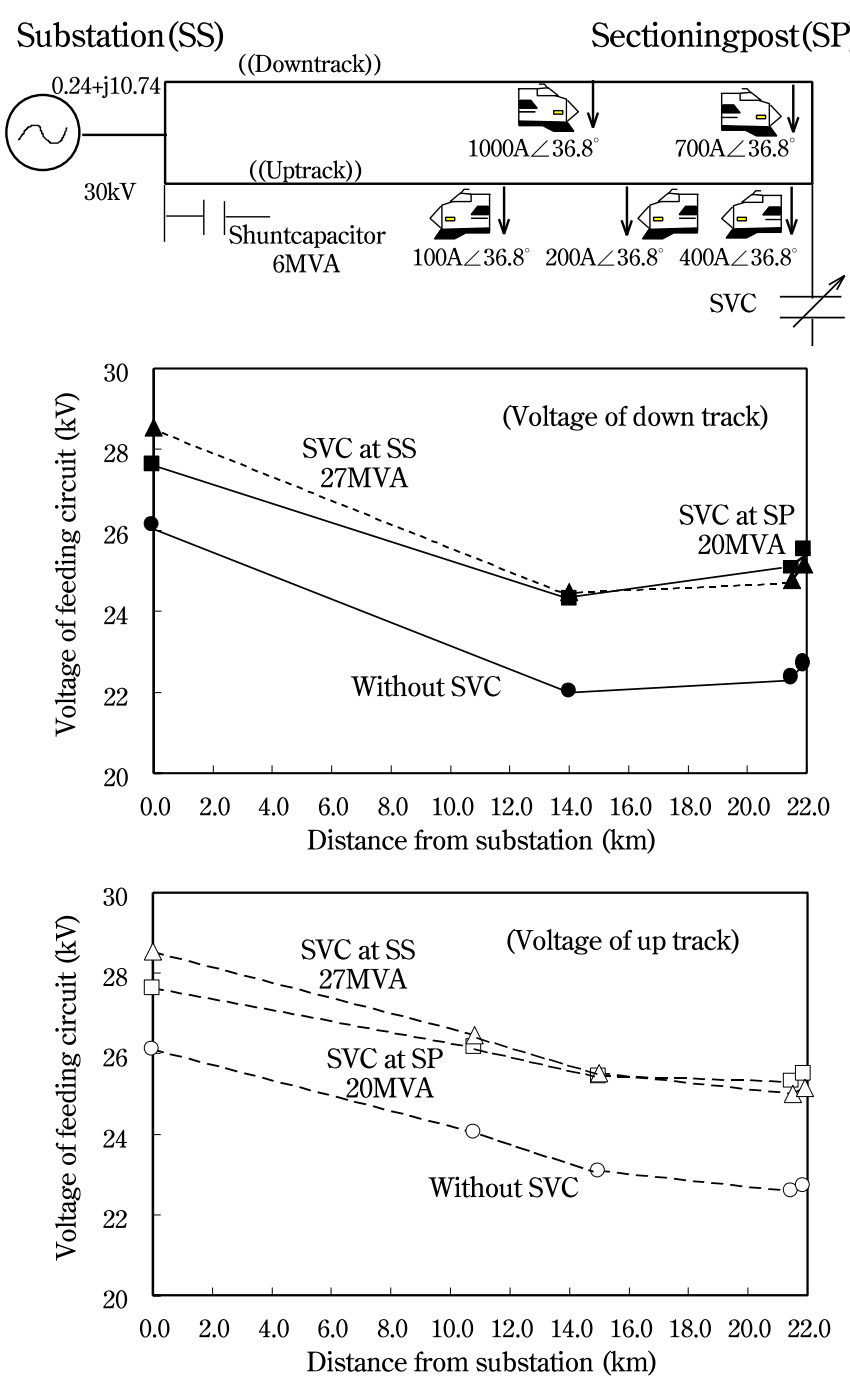

Fig. 3 Distribution of voltage

capacity of SVC can be reduced to $2 / 3$ when it is located at a sectioning post instead of at a substation.

\section{Outline of SVC at sectioning post}

\subsection{Configuration of SVC}

Fig.4 shows an overview of SVC with TSC method installed at the Shin-syo sectioning post on San-yo Shinkansen.

The electrification of San-yo Shinkansen between Shin-Osaka and Hakata is under the AT (auto-transformer) feeding system. As shown in Fig.5, the SVC is connected between the contact wire and the rail. The SVC compensates for the voltage drop by applying two banks of capacitors in order. The capacity of compensation is 15MVA in the direction of Hakata and 20 MVA in the direction of Shin-Osaka, because the train load is heavier in the direction of Shin-Osaka than in the direction of Hakata.

Table 3 shows the specification of SVC.

The high impedance transformer performs the function of suppressing the transient phenomena and fixing



Fig. 4 Overview of SVC

Substation(SS)

Sectioning post (sp)

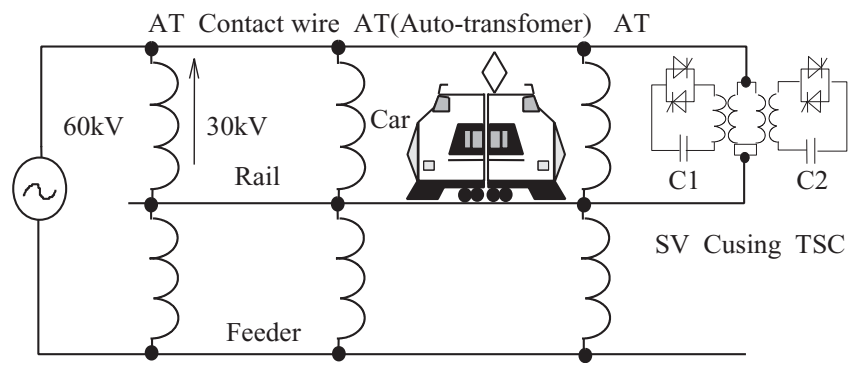

Fig. 5 AT feeding system using SVC at sectioning post

Table 3 Specification of SVC

Transformer for thyristor

\begin{tabular}{|l|c|}
\hline Number of phases & Single phase \\
Frequency & $60 \mathrm{~Hz}$ \\
Rated primary voltage & $30 \mathrm{kV}$ \\
Rated secondary voltage & $5.5 \mathrm{kV} / 5.5 \mathrm{kV}$ \\
Rated capacity & $20 / 10 / 10 \mathrm{MVA}$ \\
Impedance voltage & $15 \%$ \\
\hline
\end{tabular}

Capacitor bank and thyristor

\begin{tabular}{|l|c|}
\hline Number of phases & Single phase \\
Frequency & $60 \mathrm{~Hz}$ \\
Circuit voltage & $5000 \mathrm{~V}$ \\
Circuitcurrent & $2000 \mathrm{~A}$ \\
Capacity & $11.5 \mathrm{MVA}$ \\
L/C & $15 \%$ \\
Withstand voltage of a thyristor & $4000 \mathrm{~V}$ \\
Connection of thyristor & $9 \mathrm{~S} 1 \mathrm{P} \times 2 \mathrm{G}$ \\
\hline
\end{tabular}

its reactance so that the resonance of the third harmonic current from the train load does not spread into the feeding circuit. It also has the function to reduce the voltage on the secondary side, considering the withstand voltage of thyristors.

The cooling system for thyristors adopts the demineralized water method. The power source for the cooling system uses 3-phase power transformed by a converter from the single-phase power of the feeding circuit.

A connection diagram of the SVC is shown in Fig.6. The SVC detects the fluctuation of the feeding voltage by using the voltage sensor and operates thyristor switches 


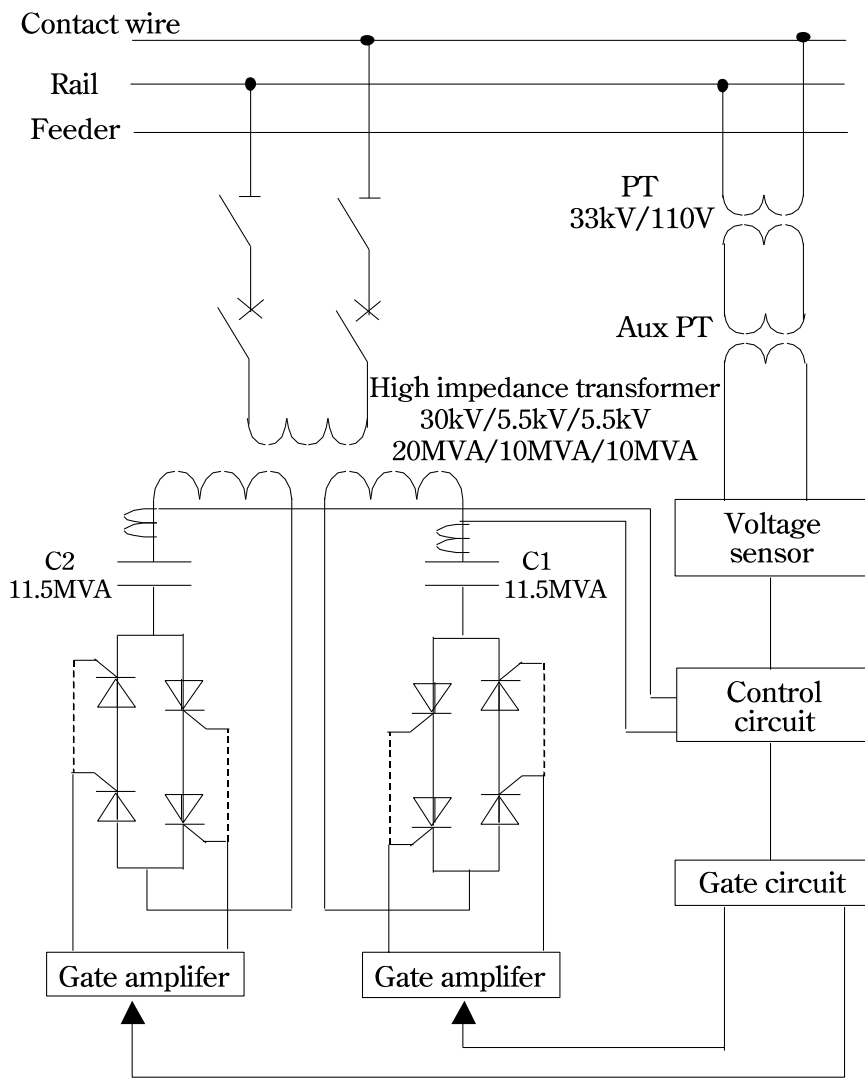

Fig. 6 SVC using TSC

by the gate circuit.

When the feeding voltage drops below the set point ( $26 \mathrm{kV}$ in Fig. 7 ), the capacitor (C1) is turned on. When the feeding voltage drops below the setting point again, the capacitor (C2) is also turned on. When the feeding voltage restores to the set point to turn off ( $29 \mathrm{kV}$ in Fig. 7 ), the capacitor is turned off.

As shown in Fig.8, the capacitor of SVC is always charged to be equal to the maximum value of feeding volt-

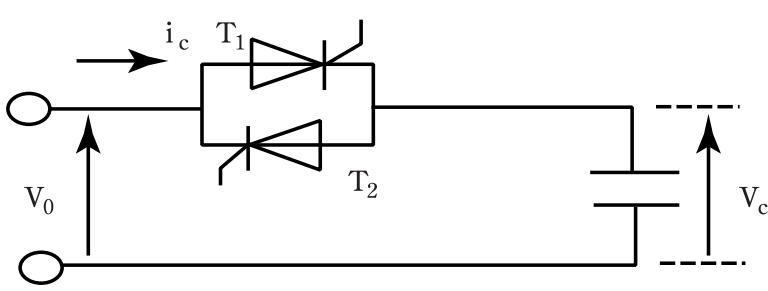

(a) Basic circuit of TSC

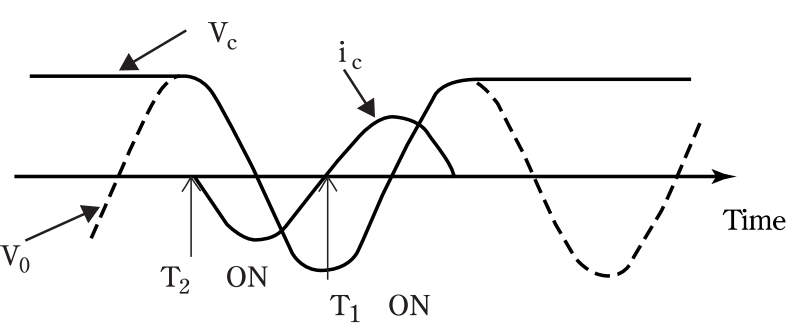

(b) Timing of turning on

Fig. 8 Method of charging maximum voltage using SVC

age by applying the thyristor (T1) as a diode, to prevent the transient phenomena when the SVC is turned on.

\subsection{Cooperation with feeding circuit and protecting system}

(1) Cooperation with feeding circuit

Because the SVC detects the fluctuation of the feeding voltage by using the voltage sensor, there is a possibility that the SVC is turned on by a transition voltage drop when a fault occurs in the feeding circuit or a train load approaches the feeding area with the SVC from the adjoining feeding area. As shown in Fig.9, the SVC is turned on in about $10 \mathrm{~ms}$ after the voltage drop is detected. If a fault occurs on the feeding circuit with SVC

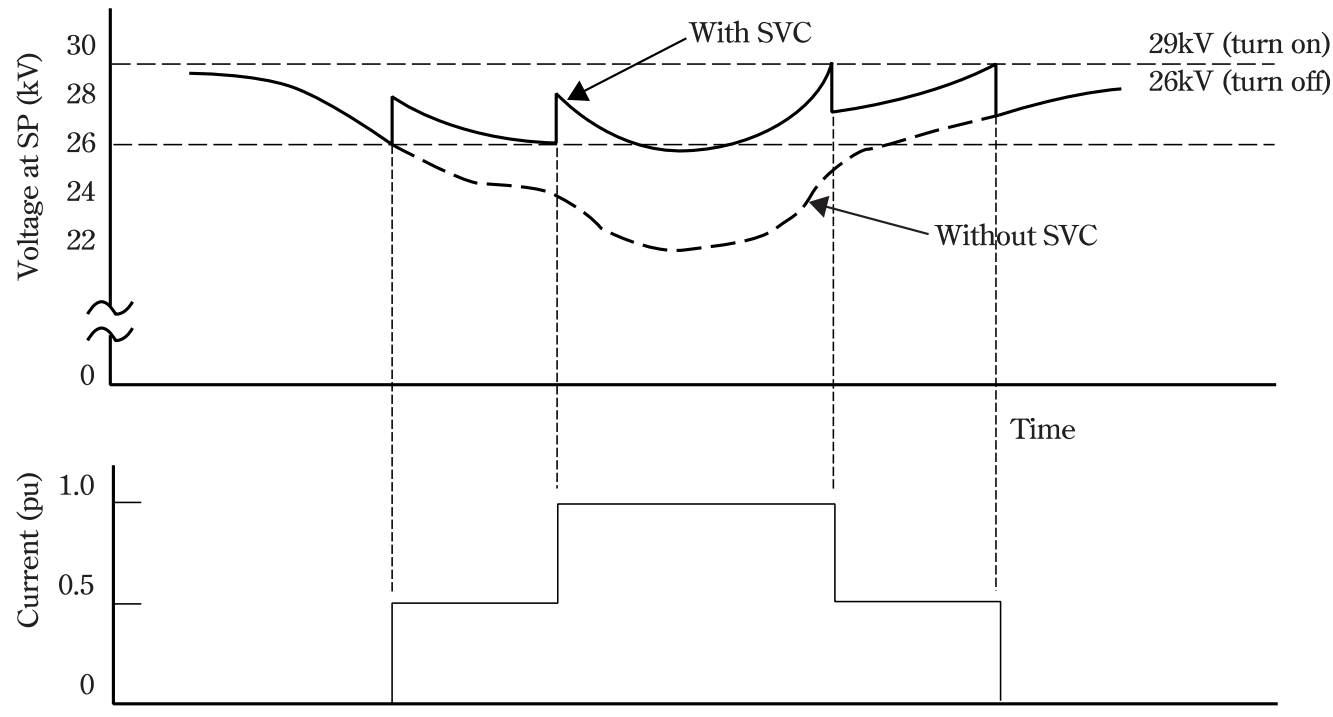

Fig. 7 Outline of voltage compensation using SVC 

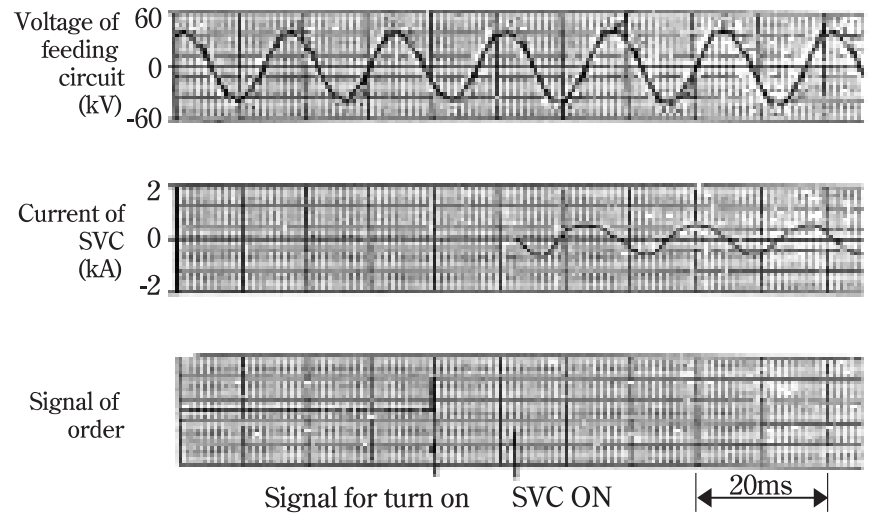

Fig. 9 Feeding voltage and current of SVC

turned on, the SVC, which is located at the sectioning post on the end of feeding circuit, is turned off immediately after the fault has occurred.

(2) Cooperation with protecting system

When the capacity of SVC is large, a fault locator, which uses the AT neutral current, accurately measures the location of the fault by AT neutral current in consideration of the SVC current.

\section{Results of measurement at actual loads}

\subsection{Effect of compensation using SVC at} sectioning post

Fig.10 shows an oscillogram of the feeding voltage at the Shin-syo sectioning post before and after the compen-

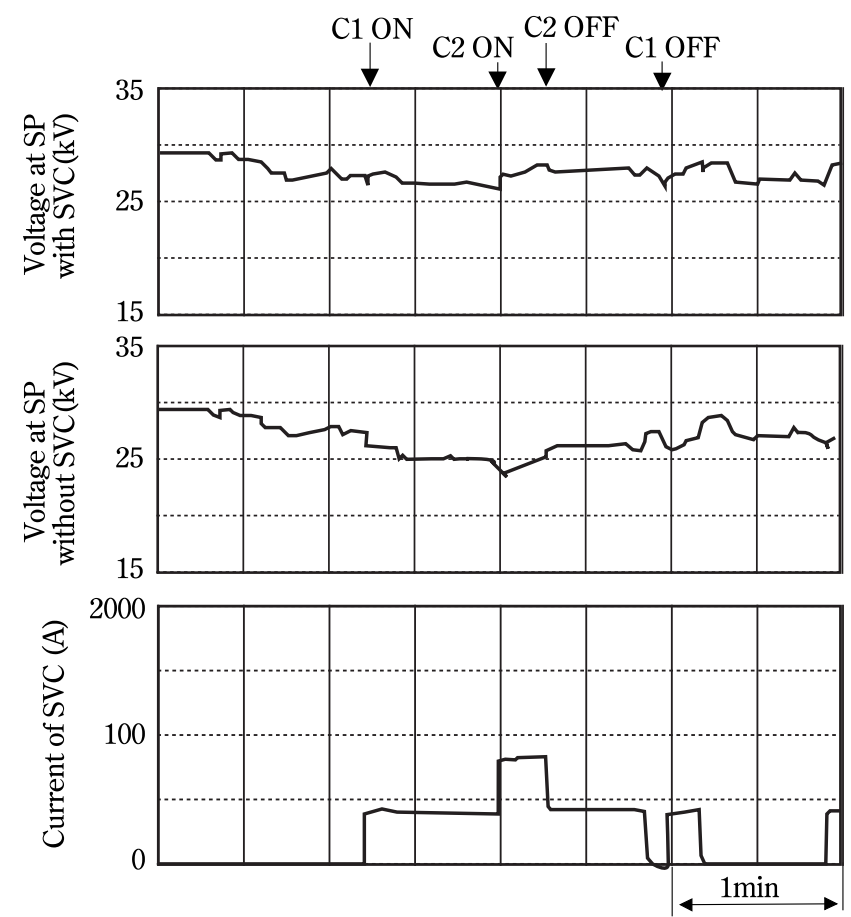

Fig. 10 Oscillogram of voltage at SP without and with SVC

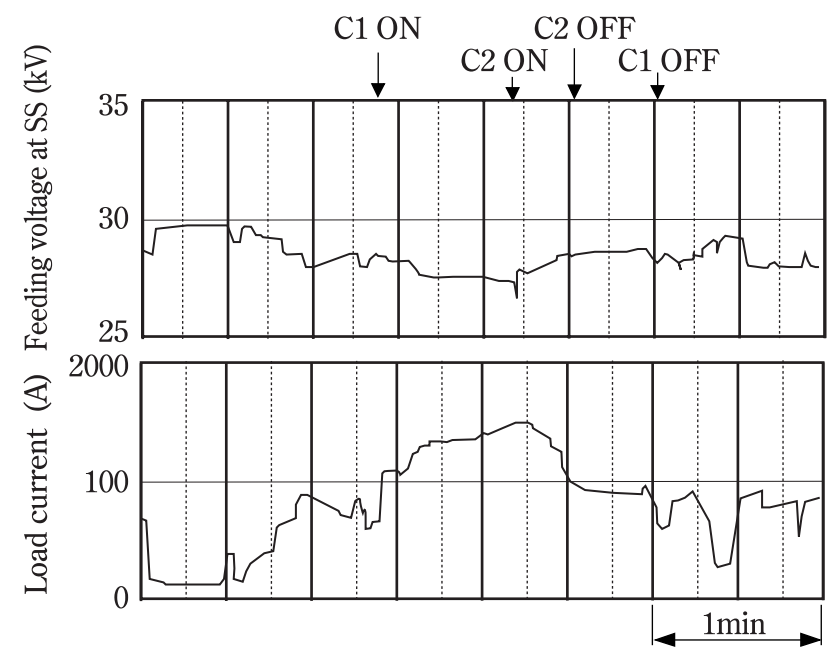

Fig. 11 Oscillogram of voltage and load current at SS

sation, and Fig.11 shows an oscillogram of the feeding voltage and load current at the substation. When the voltage in the feeding line dropped below the set point $26 \mathrm{kV}$ and rose to over $29 \mathrm{kV}$, the SVC was turned on and off, respectively. It is seen that the feeding voltage can rise about $1.5 \mathrm{kV}$ by $\mathrm{SVC}$ as a bank.

\subsection{Load zone measuring at substation}

Fig.12 shows the load zone in resistance - reactance $(R-X)$ plane of trains with SVC measuring at substation. It is confirmed from this figure that the powerfactor angle of trains is about $30 \sim 45$ degrees without SVC, which is improved when a SVC is used.

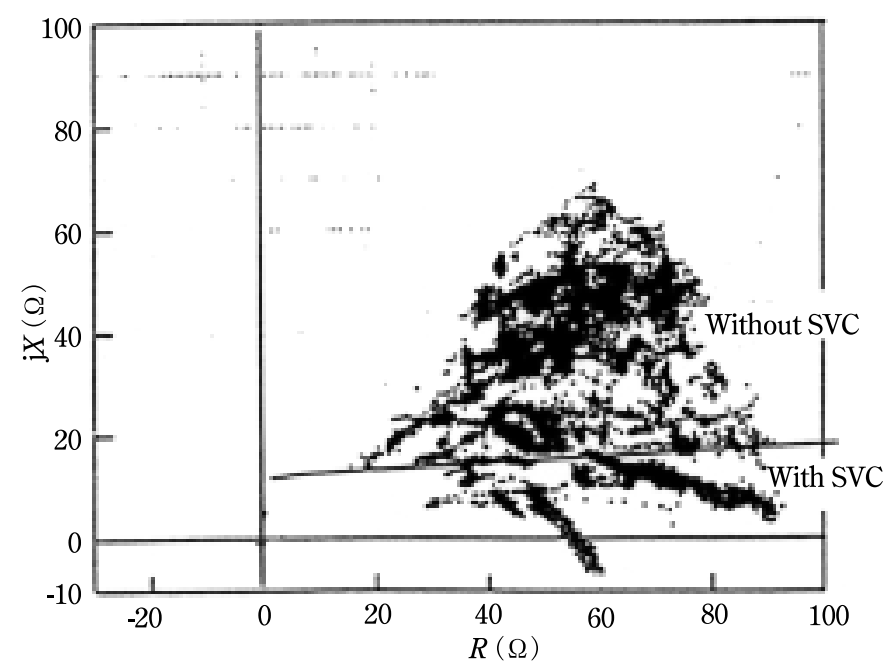

Fig. 12 Load zone in R-X plane of trains with SVC measuring at SS

\section{Conclusions}

In compensating for the voltage drop in the AC feed- 
ing system by using SVC, it is more effective to locate the SVC at a sectioning post than at a substation.

The verification site tests of the SVC with TSC at the Shin-syo sectioning post on the San-yo Shinkansen have revealed that the SVC can reliably suppress the voltage drop in the feeding circuit. It is noted that the compensation of the voltage drop using an SVC at a sectioning post contributes to the improvement of load power factor and reduction of absolute value of load current.

\section{Acknowledgment}

The authors would like to express their gratitude for the guidance and encouragement extended by the colleagues who carried out much of the experimental and constructional work.

\section{References}

1) Y.Mochinaga, M.Kayama : "Practical Use of SVC in AC Electric Railway", Transactions of IEE Japan, Vol.113-D, No.10, pp.1228, 1993

2) K.Kawahara, et al. : "Compensation of Voltage Drop using Static Var Compensator at Sectioning Post for Shinkansen Power Feeding System", Transactions of IEE Japan, Vol.119-D, No.4, pp.523-529, 1999

3) Y.Mochinaga, et al. : "Countermeasure for Voltage Drop using SVC at SP in San-yo Shinkansen”, IEE Japan, Papers of technical meeting on transportation and electric railway, TER-93-35, pp.31-40, 1993

4) Y.Mochinaga, et al. : "Countermeasure for Voltage Drop using SVC at SP in Kagoshima narrow-gauge line", National convention of IEE Japan, 1073, pp.8209, 1993 